\title{
Isolated human uterine telocytes: immunocytochemistry and electrophysiology of T-type calcium channels
}

\author{
Sanda Maria Cretoiu • Beatrice Mihaela Radu • Adela Banciu • \\ Daniel Dumitru Banciu • Dragos Cretoiu • Laura Cristina Ceafalan • \\ Laurentiu Mircea Popescu
}

Accepted: 21 August 2014 / Published online: 12 September 2014

(C) The Author(s) 2014. This article is published with open access at Springerlink.com

\begin{abstract}
Recently, telocytes (TCs) were described as a new cell type in the interstitial space of many organs, including myometrium. TCs are cells with very long, distinctive extensions named telopodes (Tps). It is suggested that TCs play a major role in intercellular signaling, as well as in morphogenesis, especially in morphogenetic bioelectrical signaling. However, TC plasma membrane is yet unexplored regarding the presence and activity of ion channels and pumps. Here, we used a combination of in vitro immunofluorescence and patch-clamp technique to characterize T-type calcium channels in TCs. Myometrial TCs were identified in cell culture (non-pregnant and pregnant myometrium) as cells having very long Tps and which were positive for CD34 and platelet-derived growth factor receptor- $\alpha$. Immunofluorescence analysis of the subfamily of T-type (transient) calcium channels $\mathrm{Ca}_{\mathrm{V}} 3.1$ and $\mathrm{Ca}_{\mathrm{v}} 3.2$ presence revealed the expression of these ion channels on the cell body and Tps of non-pregnant and pregnant
\end{abstract}

Sanda Maria Cretoiu and Beatrice Mihaela Radu have contributed equally to this article.

S. M. Cretoiu $(\bowtie) \cdot$ D. Cretoiu $\cdot$ L. C. Ceafalan

L. M. Popescu $(\square)$

Division of Cell Biology and Histology, Carol Davila University of Medicine and Pharmacy, 050474 Bucharest, Romania

e-mail: sanda@cretoiu.ro

L. M. Popescu

e-mail: LMP@ jcmm.org

S. M. Cretoiu · D. D. Banciu

Department of Ultrastructural Pathology, Victor Babeş National Institute of Pathology, 050096 Bucharest, Romania

B. M. Radu

Department of Neurological and Movement Sciences, University of Verona, 37134 Verona, Italy myometrium TCs. The expression in TCs from the nonpregnant myometrium is less intense, being confined to the cell body for $\mathrm{Ca}_{\mathrm{v}} 3.2$, while $\mathrm{Ca}_{\mathrm{v}} 3.1$ was expressed both on the cell body and in Tps. Moreover, the presence of T-type calcium channels in TCs from non-pregnant myometrium is also confirmed by applying brief ramp depolarization protocols. In conclusion, our results show that T-type calcium channels are present in TCs from human myometrium and could participate in the generation of endogenous bioelectric signals responsible for the regulation of the surrounding cell behavior, during pregnancy and labor.

Keywords Telocytes $\cdot$ Human myometrium - T-type calcium channels $\cdot \mathrm{Ca}_{\mathrm{v}} 3.1 \cdot \mathrm{Ca}_{\mathrm{v}} 3.2$

\author{
Abbreviations \\ TCs Telocytes \\ Tps Telopodes \\ SMCs Smooth muscle cells \\ PDGFR Platelet-derived growth factor receptor
}

B. M. Radu · A. Banciu - D. D. Banciu

Department of Anatomy, Animal Physiology and Biophysics,

Faculty of Biology, University of Bucharest, 050095 Bucharest,

Romania

D. Cretoiu · L. C. Ceafalan

Department of Molecular Medicine, Victor Babeş National Institute of Pathology, 050096 Bucharest, Romania

L. M. Popescu

Division of Advanced Studies, Victor Babeş National Institute of Pathology, 050096 Bucharest, Romania 
ER Endoplasmic reticulum

$\alpha$-SMA Alpha-smooth muscle actin

$\mathrm{Ca}_{\mathrm{V}} 3.1 \quad \alpha 1 \mathrm{G}$ subunit of T-type calcium channels

$\mathrm{Ca}_{\mathrm{V}} 3.2 \alpha 1 \mathrm{H}$ subunit of T-type calcium channels

LVA Low-voltage activated currents

HVA High-voltage activated currents

\section{Introduction}

Regulation of contractile activity of the uterus is an important process, and numerous studies aimed to determine the mechanism of uterine activation during term and preterm parturition (Aguilar and Mitchell 2010). Hitherto, little is known about the physiological triggers of uterine contraction, and the roles of interstitial cells and matrix are frequently neglected (Hutchings et al. 2009). However, there are some new cellular elements in the interstitial spacethe telocytes (TCs) - that seem to be important (jointly with other connective tissue cells) for the normal functionality of mammalian organs (Zheng et al. 2012, 2013; Cretoiu et al. 2012a; Mou et al. 2013; Corradi et al. 2013; Díaz-Flores et al. 2013; Matyja et al. 2013; Luesma et al. 2013) including human uterus (Popescu et al. 2006b, 2007; Cretoiu et al. 2011). TCs have a small cell body with very long distinctive prolongations- - the telopodes (Tps) (Popescu and Faussone-Pellegrini 2010). A TC can have one up to five Tps with alternating podoms (dilated segments) and podomers (thin segments). Podoms offer accommodation for mitochondria, ER and caveolae, a trio involved in calcium homeostasis (Gherghiceanu and Popescu 2012). Tps have a dichotomous branching pattern, building a 3D network due to homocellular junctions (Ceafalan et al. 2012; Cretoiu et al. 2012b), and occupy a strategic position in relation to stem cell niches, blood capillaries and/or nerve bundles (Gherghiceanu and Popescu 2011; Popescu and Nicolescu 2013). Heterocellular nanocontacts were frequently described between TCs and myocytes (Gherghiceanu and Popescu 2011; Cretoiu et al. 2012a) or TCs and immune cells (Popescu et al. 2005). TCs were shown to release ectosomes and/or exosomes suggesting a role in intercellular signaling (Cretoiu et al. 2013). The immunohistochemical profile of TCs is not yet defined by specific markers; however, several studies claim that TCs are frequently found to be positive for CD34 and PDGFR $\alpha$ or PDGFR $\beta$ within the connective tissue in mucosa and submucosa of different organs or in the interstitium of the muscular coat of cavitary organs (Vannucchi et al. 2013; Chen et al. 2013; Milia et al. 2013; Xiao et al. 2013), as well as for Oct4 in mouse lung (Galiger et al. 2014). The gene profile of TCs was reported (Zheng et al. 2013) in mouse lung, as well as patterns of mouse TC-specific gene profiles on chromosome 1 (Sun et al. 2014). The TCs proteome certified that indeed, TCs are different from fibroblasts and from endothelial cells (Zheng et al. 2014a, b).

Electrophysiologically, there is evidence about the fact that atrial and ventricular TCs present large conductance $\mathrm{Ca}^{2+}$-activated $\mathrm{K}^{+}$currents and inwardly rectifying $\mathrm{K}^{+}$ currents, but not transient outward $\mathrm{K}^{+}$-currents and ATPsensitive potassium currents (Sheng et al. 2014). Moreover, in human uterus, small-conductance calcium-activated potassium currents have been detected (Rosenbaum et al. 2012), and preliminary in vitro electrophysiological evaluation of the TCs in non-pregnant uterus revealed a hyperpolarization-activated chloride inward current with calcium dependence (Cretoiu et al. 2013).

The subfamily of T-type (transient) calcium channels consists of several different subunits: $\alpha_{1}, \alpha_{2} \delta, \beta_{1-4}$ and $\gamma$. Differences in molecular structure of $\alpha_{1}$ subunits subdivided them as $\mathrm{Ca}_{\mathrm{V}} 3.1\left(\alpha_{1 \mathrm{G}}\right), \mathrm{Ca}_{\mathrm{V}} 3.2\left(\alpha_{1 \mathrm{H}}\right)$ and $\mathrm{Ca}_{\mathrm{V}} 3.3\left(\alpha_{1 \mathrm{I}}\right)$. These subunits are most often found in cells that have pacemaker activity (Blanks et al. 2007; Senadheera et al. 2013). Although T-type calcium channels have been extensively studied in human myometrium (Young et al. 1993; Young and Zhang 2005), no evidence was yet documented in uterine TCs.

Our purpose was to determine whether such T-type channels are present in TCs and to study whether there are any differences between the TCs in non-pregnant and pregnant myometrium. $\mathrm{Ca}_{\mathrm{v}} 3.1$ and $\mathrm{Ca}_{\mathrm{v}} 3.2$ were expressed in TCs, on the cell body and in Tps, and found to be less intense in TCs in non-pregnant myometrium. This study provides direct immunofluorescence and electrophysiological evidence for the existence of T-type calcium channels in TCs from human myometrium.

\section{Materials and methods}

Human tissue samples

Biopsies of human myometrium were obtained from two groups of women ( $n=8$, in each group) under non-pregnant and pregnant state. All tissue samples were obtained in accordance with a protocol approved by the local Bioethics Committee of the University of Medicine and Pharmacy, Bucharest, in accordance with The Code of Ethics of the Declaration of Helsinki. Informed written consent was received from all patients donating tissue samples. None of the subjects were under regular medication for chronic diseases. Non-pregnant myometrium strips were removed from the uterine supraisthmic region from hysterectomy specimens (benign conditions) of premenopausal women. Small strips of pregnant myometrium (between 38 and 40 weeks of gestation) were taken during Caesarean sections, from the upper margin (in the midline) of the lower segment transverse incision. 


\section{Cell cultures}

Human myometrial samples were collected into sterile tubes containing Dulbecco's Modified Eagle's Medium (DMEM), supplemented with fetal bovine serum (FBS) $2 \%$, HEPES $(1.5 \mathrm{mM})$ as well as $200 \mathrm{IU} / \mathrm{ml}$ penicillin, $200 \mathrm{UI} / \mathrm{ml}$ streptomycin and $0.50 \mu \mathrm{g} / \mathrm{ml}$ amphotericin B (Fungizone) (all from Gibco/Life Technologies Ltd., Paisley, UK), placed on ice and transported to the cell culture laboratory. Samples were processed within $30 \mathrm{~min}$ from surgery and rinsed with sterile DMEM. The myometrial samples were dissected under a stereomicroscope and minced into small pieces of about $1 \mathrm{~mm}^{3}$, subsequently washed and incubated with gentle agitation for $30 \mathrm{~min}$, at $37^{\circ} \mathrm{C}$, with collagenase Ia (Sigma-Aldrich, St. Louis, MO, USA) $10 \mathrm{mg} / \mathrm{ml}$ and DNAase I $(0.1 \mathrm{~nm} / \mathrm{mg})$ in DMEM supplemented with FBS $10 \%$, HEPES $1.5 \mathrm{mM}, 100 \mathrm{IU} / \mathrm{ml}$ penicillin, $100 \mathrm{UI} / \mathrm{ml}$ streptomycin and $0.25 \mu \mathrm{g} / \mathrm{ml}$ fungizone. The dispersed cells were separated from non-digested tissue by filtration through a cell strainer $(100 \mathrm{~mm})$, collected by centrifugation of the filtrate at $250 \mathrm{~g}$ for $10 \mathrm{~min}$, at room temperature $\left(22{ }^{\circ} \mathrm{C}\right)$ and suspended in culture medium. Cells were distributed in $25-\mathrm{cm}^{2}$ plastic culture flasks (BD Falcon, San Jose, CA, USA) or on glass coverslips into 24-well plates (BD Labware, San Jose, CA, USA) at a density of $5 \times 10^{4}$ cells $/ \mathrm{cm}^{2}$. Medium was changed every $48 \mathrm{~h}$. Cells were maintained at $37{ }^{\circ} \mathrm{C}$ in a humidified atmosphere $\left(5 \% \mathrm{CO}_{2}\right.$ in air) until becoming semi-confluent (usually 4 days after plating) when the cells were detached using $0.25 \%$ trypsin and $2 \mathrm{mM}$ EDTA and replated at the same density of $5 \times 10^{4}$ cells $/ \mathrm{cm}^{2}$. Experiments were performed between passages 1 and 4. Cells were examined and photographed under a Nikon inverted TE200 microscope equipped with a Nikon DN-5 digital camera.

\section{Immunocytochemistry}

To visualize cell expressing T-type calcium channels, immunofluorescent labeling was performed on cells grown on coverslips (Ciontea et al. 2005). Cell cultures were obtained from both non-pregnant and pregnant myometrium. Samples were fixed in $2 \%$ paraformaldehyde for $10 \mathrm{~min}$, washed in PBS and then incubated in PBS containing $2 \%$ bovine serum albumin (BSA) for another $10 \mathrm{~min}$. Next, cells were washed and permeabilized with $0,075 \%$ saponin in PBS for 10 min (all reagents from Sigma-Aldrich, St. Louis, MO, USA). Incubation with the primary antibodies was performed at room temperature for $1 \mathrm{~h}$ using antihuman antibodies with the listed specificities and working dilutions (Table 1). Primary antibodies were detected with secondary anti-mouse antibody conjugated to AlexaFluor 546, 1:300 or AlexaFluor488, 1:300; secondary goat anti-rabbit antibody
Table 1 Panel of antibodies

\begin{tabular}{llll}
\hline Antibody & Code no. & Host species & Dilution \\
\hline T-type $\mathrm{Ca}^{2+} \mathrm{CP} \alpha 1 \mathrm{H}$ & sc-377510 & Mouse & $1: 150$ \\
T-type $\mathrm{Ca}^{2+} \mathrm{CP} \alpha 1 \mathrm{G}$ & sc-28617 & Rabbit & $1: 200$ \\
CD34 & sc-7045 & Goat & $1: 50$ \\
Smooth muscle actin Ab-1 & MS-113-P & Mouse & $1: 200$ \\
PDGFR $\alpha$ & sc-338 & Rabbit & $1: 100$ \\
\hline
\end{tabular}

conjugated to AlexaFluor 488, 1:250; donkey anti-goat antibody conjugated to AlexaFluor 546, 1:250 all from Invitrogen Molecular Probes, Eugene, OR, USA. Nuclei were finally counterstained with $1 \mu \mathrm{g} / \mathrm{ml} 4^{\prime}$,6-diamidino-2-phenylindole (DAPI) (Sigma-Aldrich). Negative controls were obtained following the same protocol, but omitting the primary antibodies. Samples were examined under a Nikon TE300 microscope equipped with a Nikon DS-Qi1 camera, Nikon PlanApo $20 \times$ and $40 \times$ objectives, and the appropriate fluorescence filters. The immunolabelled samples were photographed, and images were randomly selected and evaluated for fluorescent intensity analysis. The images were loaded into Image J, the region of interest (ROI) traced and the 'gray level intensity' analyzed. Background gray level intensity was also measured and subtracted for each image (Burgess et al. 2010; Radu et al. 2014). Resulting data were statistically analyzed using Microsoft Excel analysis tool pack.

\section{Patch-clamp recordings}

TCs from non-pregnant uterus were recorded in wholecell configuration under the voltage-clamp mode, using an AxonPatch 200B amplifier (Molecular Devices, USA). Electrodes were pulled from borosilicate glass capillaries (GC150F; Harvard Apparatus, Edenbridge, Kent, UK) and heat polished. The final resistance of the pipette, when filled with internal solution, was 3-4 M $\Omega$. The perfusion was performed with an MPS-2 (World Precision Instruments, Sarasota, FL, USA) system, with the tip placed at approximately $100 \mu \mathrm{m}$ from the cell. Membrane currents were low-pass filtered at $3 \mathrm{kHz}(-3 \mathrm{~dB}, 3$ pole Bessel) and sampled with a Axon Digidata 1440 data acquisition system (Molecular Devices, USA) using pClamp 10 software in gap-free mode. All electrophysiological experiments were performed at room temperature $\left(25^{\circ} \mathrm{C}\right)$.

The bath solution contained $(\mathrm{mM})$ : tetraethylammonium (TEA)-Cl 130, $\mathrm{BaCl}_{2} 10, \mathrm{MgCl}_{2}$ 1, HEPES 10 , glucose 10 , adjusted to $\mathrm{pH} 7.4$ at $25^{\circ} \mathrm{C}$ with TEA-OH. The pipette solution contained (mM): $\mathrm{CsCl} 137 ; \mathrm{MgCl}_{2} 1$; HEPES 10; 1,2-bis(2-aminophenoxy)ethane- $N, N, N^{\prime}, N^{\prime}$-tetraacetic acid (BAPTA) 10; Mg-ATP 1, adjusted to pH 7.3 at $25{ }^{\circ} \mathrm{C}$ with $\mathrm{CsOH}$. Solutions for T-type calcium channel recordings 
have been used as previously described (Comunanza et al. 2011), and only EGTA was replaced by BAPTA in the pipette filling solution in order to increase the stability of the baseline.

Two voltage protocols have been applied on TCs from non-pregnant uterus in order to evoke T-type calcium currents: (1) step depolarization protocol with pulses (from -90 to $+40 \mathrm{mV}$ ) of $100 \mathrm{~ms}$ duration, $10-\mathrm{mV}$ increment from a holding potential of $-110 \mathrm{mV}$ as previously described (Ohkubo et al. 2005) and (2) brief depolarizing ramp protocol from -90 to $+60 \mathrm{mV}$ with a duration of $100 \mathrm{~ms}(1.5 \mathrm{~V} / \mathrm{s})$, as previously described (Comunanza et al. 2011). Mibefradil $(1 \mu \mathrm{M})$, a potent antagonist for T-type calcium channels (Clozel et al. 1997; Leuranguer et al. 2001), was applied 4 min before and during the brief depolarization ramp protocols.

In order to test whether the calcium current has a steadystate component, we have applied ramp depolarization protocols with different slopes 0.5 and $0.05 \mathrm{~V} / \mathrm{s}$, in a similar manner as the protocol previously described (Baruscotti et al. 2000).

Data were analyzed in Clampfit 10.2 (Molecular Devices Corporation, USA) and plotted in Origin 8.6. Statistical analysis was done by paired Student's $t$ test, and data are presented as mean $\pm \mathrm{SD}$.

\section{Results}

TCs identification in myometrial cell cultures

Under phase-contrast microscopy, in primary cell cultures, TCs were easily distinguished from smooth muscle cells (SMCs) before cell confluence. According to previous studies cell cultures derived from non-pregnant and pregnant myometrium, tissue samples are able to maintain the cell phenotype up to the tenth passage (Leoni et al. 1990; Mosher et al. 2013) and validated primary cell culture usefulness for studying pregnancy and labor (Mosher et al. 2013). In both type of cultures, from non-pregnant and pregnant myometrium, TCs displayed the characteristic silhouette and extend between 1 and 3 long, moniliform Tps (Fig. 1a, e). In order to analyze whether these cells are indeed TCs, we performed immunofluorescence for CD34 and PDGFR $\alpha$ antigens. Immunostaining of non-pregnant and pregnant myometrial cells in culture revealed the presence of TCs as CD34/PDGFR $\alpha$-positive cells (Fig. 1b, c, $\mathrm{f}, \mathrm{g}$ ), corresponding to the phenotype described by others in situ (Vannucchi et al. 2013; Milia et al. 2013, Xiao et al. 2013; Qi et al. 2012) or in vitro (Mou et al. 2013). Double immunofluorescence staining against CD34 and PDGFR $\alpha$ revealed intense CD34 immunostaining at Tps level, while
PDGFR $\alpha$ was expressed mostly in the cell body as it can be observed on merged images (Fig. 1d, h).

Immunofluorescence for T-type calcium channels in TCs from human myometrium

We also investigated the expression of $\alpha$-subunits $\mathrm{Ca}_{\mathrm{V}} 3.1$ $\left(\alpha_{1 \mathrm{G}}\right)$ and $\mathrm{Ca}_{\mathrm{V}} 3.2\left(\alpha_{1 \mathrm{H}}\right)$ by immunofluorescence microscopy in cell cultures. Immunohistochemical staining using anti-Ca 3.1 and anti-Ca 3.2 antibodies showed the expression of $\alpha_{1 \mathrm{G}}$ and $\alpha_{1 \mathrm{H}}$ in both non-pregnant and pregnant myometrial cell cultures (Fig. 2). $\mathrm{Ca}_{\mathrm{V}} 3.1\left(\alpha_{1 \mathrm{G}}\right)$ and $\mathrm{Ca}_{\mathrm{V}} 3.2$ $\left(\alpha_{1 \mathrm{H}}\right)$ reactivity was observed in cells with morphologies suggestive for TCs. In non-pregnant myometrial cell cultures, T-type voltage-dependent calcium channel $\mathrm{Ca}_{\mathrm{v}} 3.1$ $\left(\alpha_{1 \mathrm{G}}\right)$ and $\mathrm{Ca}_{\mathrm{V}} 3.2\left(\alpha_{1 \mathrm{H}}\right)$ isoforms revealed differences in their localization: The intensity of $\mathrm{Ca}_{\mathrm{v}} 3.1$ immunostaining was stronger at Tps level (Fig. 2a), while $\mathrm{Ca}_{\mathrm{v}} 3.2$ was expressed only on the cell body (Fig. 2b). Double-labeling immunofluorescence methods were used to provide evidence that both isoforms were expressed in the same TC (Fig. 2c). In pregnant myometrial cell cultures, strong staining for $\mathrm{Ca}_{\mathrm{V}} 3.1$ (Fig. 2d) and $\mathrm{Ca}_{\mathrm{V}} 3.2$ (Fig. 2e) was found in the cell body of TCs and in the Tps (Fig. 2f) coexisting in the same cell. We semi-quantitatively evaluated the intensity of the fluorescence for $\mathrm{Ca}_{\mathrm{v}} 3.1$ and $\mathrm{Ca}_{\mathrm{v}} 3.2$ in both SMCs and TCs and also compared the fluorescence intensity in TCs derived from non-pregnant and pregnant myometrium (Fig. 3).

Figure 4 summarizes that at least two types of cells were present in culture: (a) TCs with distinct morphologies and positive for $\mathrm{Ca}_{\mathrm{v}} 3.1$ and (b) SMC with widened cell body without extensions, positive for $\alpha$-smooth muscle actin ( $\alpha$-SMA). TCs were also positive for $\alpha$-SMA, but the immunoreactivity was very weak and actin filaments are homogenously distributed and do not form stress fibers like those known to exist in cultured SMCs (Deguchi et al. 2006; Matsumoto and Nagayama 2012). Furthermore, we showed that both cell types (TCs and SMC) were positive for $\mathrm{Ca}_{\mathrm{v}} 3.1$ and $\mathrm{Ca}_{\mathrm{v}} 3$ 3.2. In cell cultures, from both nonpregnant and pregnant myometrium, $\mathrm{Ca}_{\mathrm{V}} 3.2$ expression in TCs was weaker compared with $\mathrm{Ca}_{\mathrm{v}} 3.1$. However, the expression level of $\mathrm{Ca}_{\mathrm{v}} 3.1$ was higher in TCs from pregnant myometrium compared with TCs from non-pregnant myometrium.

Electrophysiology of T-type calcium channels in TCs from human myometrium

The presence of T-type calcium channels in TCs was tested by the patch-clamp technique (Fig. 5a) in voltage-clamp mode, using brief ramp depolarization protocol (Fig. 5b, 

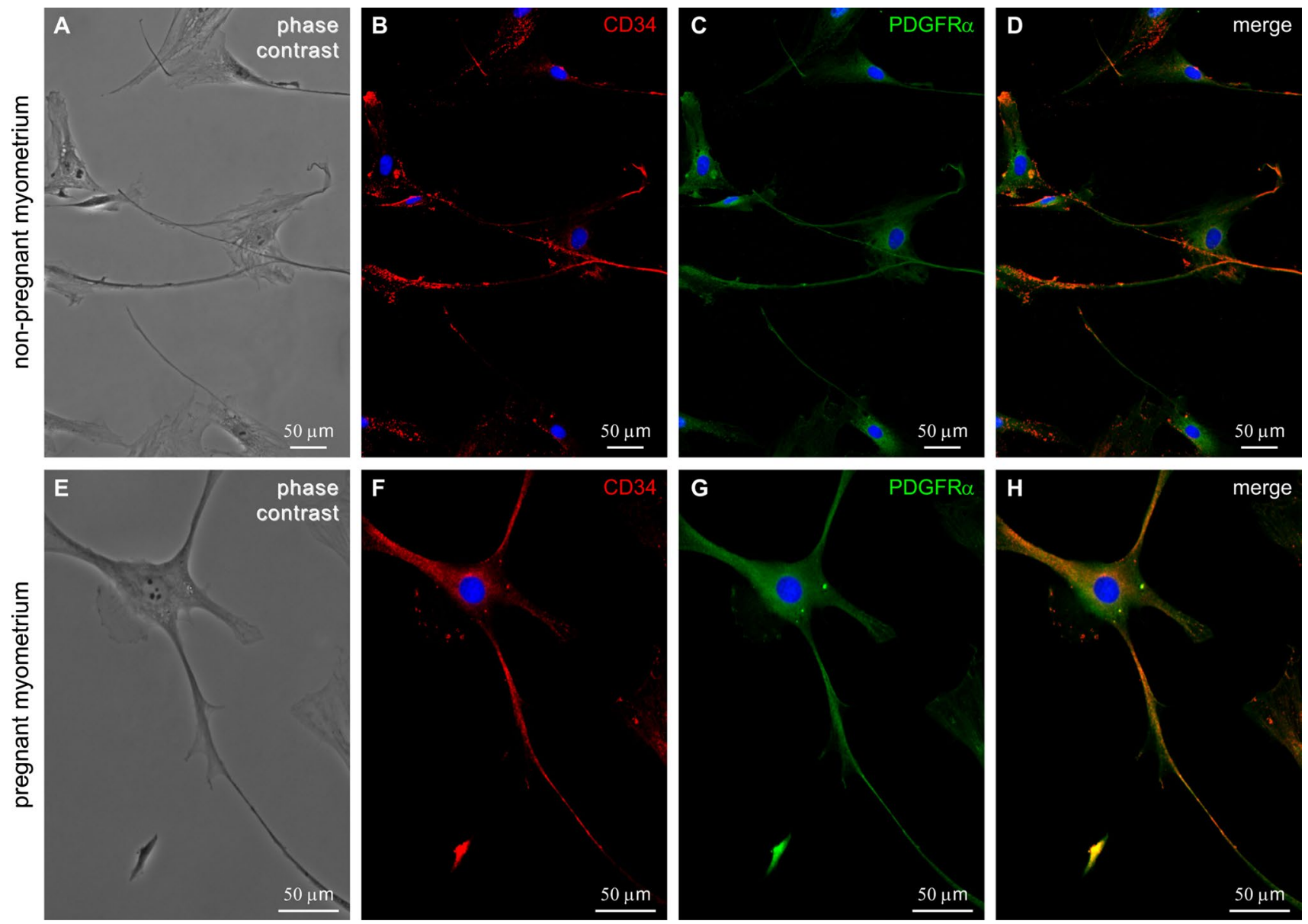

Fig. 1 TCs in cell cultures from human non-pregnant/pregnant myometrium after $72 \mathrm{~h}$ in culture at first passage. a, e Phase-contrast microscopy depicts cell morphologies very evocative for TCs: small cell body with very long Tps characterized by a moniliform silhouette (alternation of podoms and podomers). Fluorescence microscopy

upper insert) and step-depolarizing pulse protocol (Fig. 5c, upper insert).

A standard protocol of step-depolarizing pulses (from -90 to $+40 \mathrm{mV}$ ) in $10-\mathrm{mV}$ increments of $100 \mathrm{~ms}$ duration from a holding potential of $-110 \mathrm{mV}$ (Fig. 5b) did not evoke any T-type calcium current neither in TCs $(n=12)$.

Brief ramp depolarization protocol from -90 to $+60 \mathrm{mV}$ with duration of $100 \mathrm{~ms}$ evoked two types of calcium currents (Fig. 5c), T-type calcium current [low-voltage activated calcium currents (LVA)] and high-voltageactivated calcium currents (HVA), as previously described by Comunanza et al. (2011). T-type calcium channels were activated at $-50 \mathrm{mV}$. By applying $1 \mu \mathrm{M}$ mibefradil, we have obtained the amplitude reduction of the T-type calcium current from $178 \pm 47 \mathrm{pA}(n=4)$ to $98 \pm 22 \mathrm{pA}$ ( $n=4, p<0.05$, paired Student's $t$ test), and of the HVA current from $100 \pm 25 \mathrm{pA}(n=4)$ to $57 \pm 28 \mathrm{pA}(n=4$, shows CD34 (b, f) and PDGFR- $\alpha$ (c, g)-positive TCs. d, h Double labeling for both markers showing a different expression pattern for PDGFR- $\alpha$ and CD34 in the same TC. Nuclei were stained with DAPI. Bar $50 \mu \mathrm{m}$

not significant, paired Student's $t$ test) represented in Fig. 5D. The HVA current was not further characterized. We have obtained an inhibitory effect of mibefradil on HVA currents (e.g., L-type calcium currents), in agreement with previous reports describing mibefradil as a partial antagonist of these channels (Leuranguer et al. 2001).

There is no steady-state component of the calcium currents. Indeed, the currents are abolished at the slowest slope of the ramp depolarization protocol (data not shown).

\section{Discussion}

Human myometrium exhibits in vitro spontaneous contractions which can be influenced by multiple factors (Hutchings et al. 2009; Cretoiu et al. 2011), including the interrelation between SMCs and TCs. Previous studies revealed that 

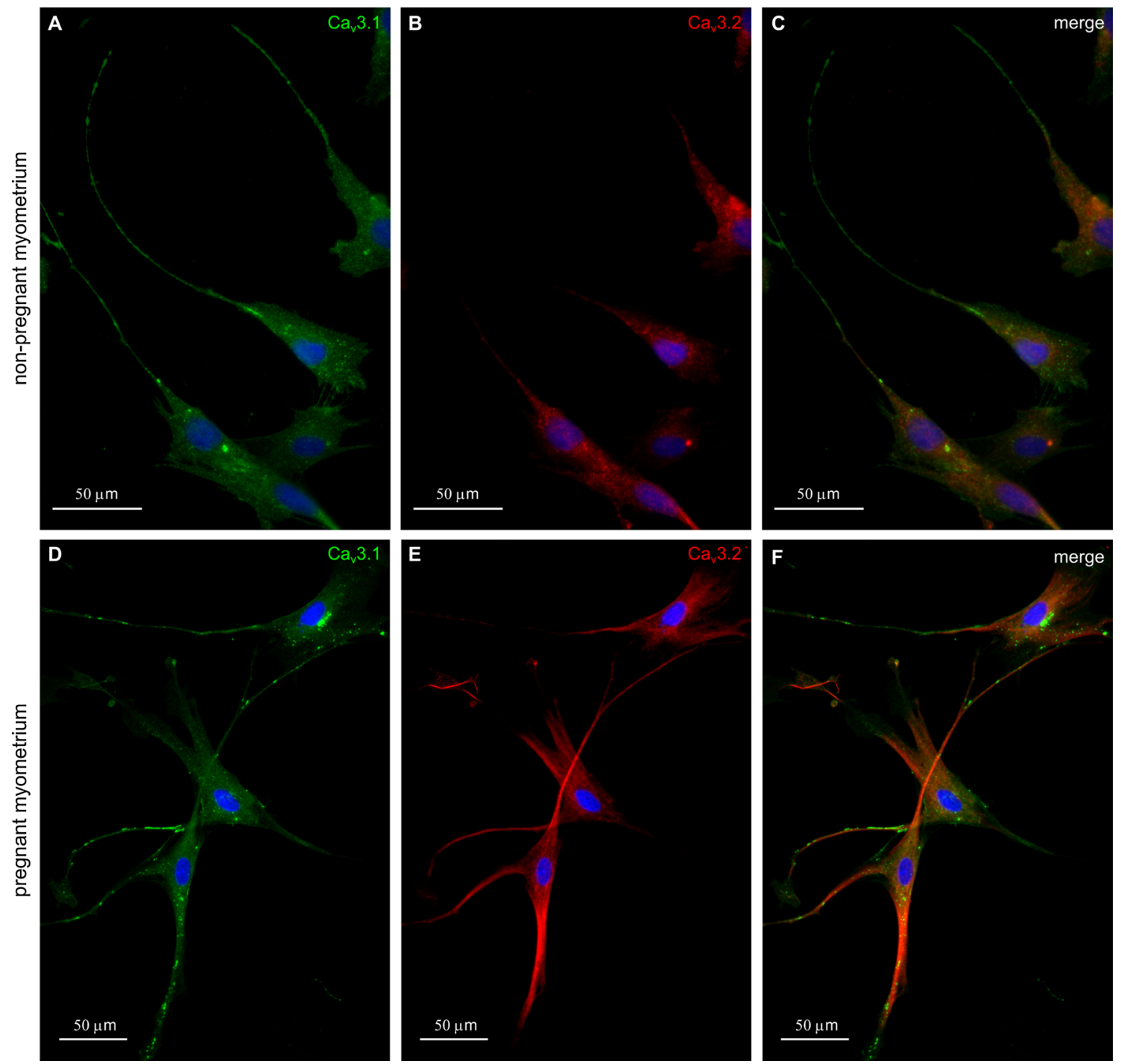

Fig. 2 Double immunolabeling for T-type calcium channels in cell cultures from non-pregnant/pregnant myometrium. a $\mathrm{Ca}_{\mathrm{V}} 3.1 \mathrm{immu}-$ nolabeling (green) was detected on TCs cell body, but it was stronger at Tps level, b $\mathrm{Ca}_{\mathrm{v}} 3.2$ expression was detected only at cell body level. c Co-expression of $\mathrm{Ca}_{\mathrm{V}} 3.1$-positive (green) and $\mathrm{Ca}_{\mathrm{V}} 3.2$ (red) is presented on merged images. d Immunolabeling for T-type calcium channels in cell cultures from pregnant myometrium. Strong staining

TCs can communicate with neighboring muscle cells possibly by gap junctions (Ciontea et al. 2005; Popescu et al. 2006a; Gherghiceanu and Popescu 2011) or by close contacts (Cretoiu et al. 2012a). Recently, it has been suggested that there is a mutual influence between TCs and SMCs acting probably by paracrine mechanisms due to exosome/ectosome release (Cretoiu et al. 2013). However, we assume that, in the myometrium, some other influences could be involved, like, as yet unknown, pathways involving the effects of female sex steroids on the regulation of TCs activity and/or interrelation with SMCs, as suggested by our previous studies (Cretoiu et al. 2006, 2009; Bassotti et al. 2013). for $\mathrm{Ca}_{\mathrm{V}} 3.1$ was found in the cytoplasm, adjacent to the nucleus and in the Tps of the TCs. e Ca 3.2 immunolabeling was found throughout the cytoplasm of TCs and predominantly within Tps. f Merged images show co-expression pattern for $\mathrm{Ca}_{\mathrm{v}} 3.1$ (green) and $\mathrm{Ca}_{\mathrm{v}} 3.2$ (red). Note that both TCs and SMCs (cells with widened cell body without extensions) express $\mathrm{Ca}_{\mathrm{v}} 3.1$ and $\mathrm{Ca}_{\mathrm{v}} 3.2$. Nuclei were stained with DAPI. Bar $50 \mu \mathrm{m}$

Our study brings evidence on $\mathrm{Ca}_{\mathrm{v}} 3.1$ and $\mathrm{Ca}_{\mathrm{v}} 3.2$ expression in uterine TCs by immunofluorescence staining. Indeed, we have detected the $\alpha$-subunit of T-type calcium channels in TCs from non-pregnant and pregnant myometrium, on the cell body and in Tps. The expression was less intense in TCs from non-pregnant myometrium. It is an interesting result, as previous immunohistochemistry, qRT-PCR and electrophysiological studies were exclusively dedicated to the $\mathrm{Ca}_{\mathrm{V}} 3.1$ and $\mathrm{Ca}_{\mathrm{V}} 3.2$ expression or T-type currents presence in SMCs from human myometrium (Young et al. 1993; Young and Zhang 2005; Ohkubo et al. 2005; Blanks et al. 2007). 
Fig. 3 Semi-quantitative analysis of fluorescence intensity of $\mathrm{Ca}_{\mathrm{V}} 3.1$ and $\mathrm{Ca}_{\mathrm{V}} 3.2$ on TCs from non-pregnant and pregnant myometrium. Roughly, one can observe that the level of fluorescence intensity is around 50 or below for SMCs, while in TCs the level is above this value. Histogram depicts that $\mathrm{Ca}_{\mathrm{V}} 3.1$ is expressed approximately equal on TCs in non-pregnant versus pregnant myometrium. Instead, $\mathrm{Ca}_{\mathrm{V}} 3.2$ is expressed more in the TCs of the pregnant myometrium versus non-pregnant myometrium. The fluorescence intensity was higher at the level of the Tps for both $\mathrm{Ca}_{\mathrm{V}} 3.1$ and $\mathrm{Ca}_{\mathrm{v}} 3.2$, in non-pregnant and pregnant myometrium

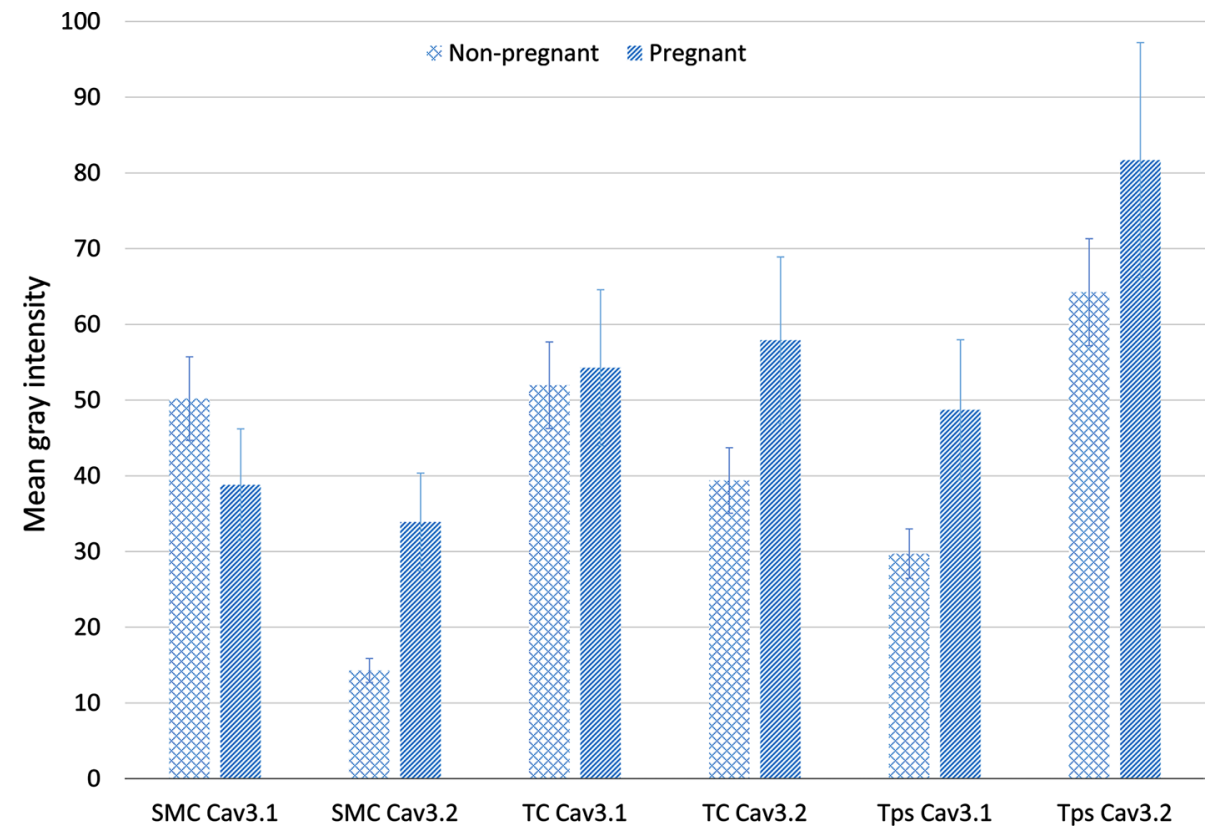

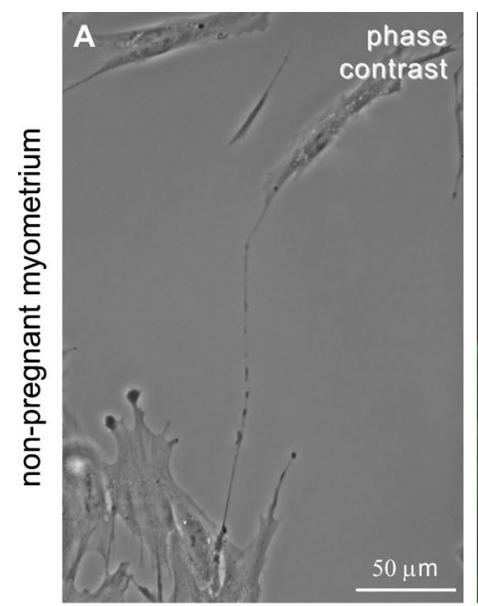
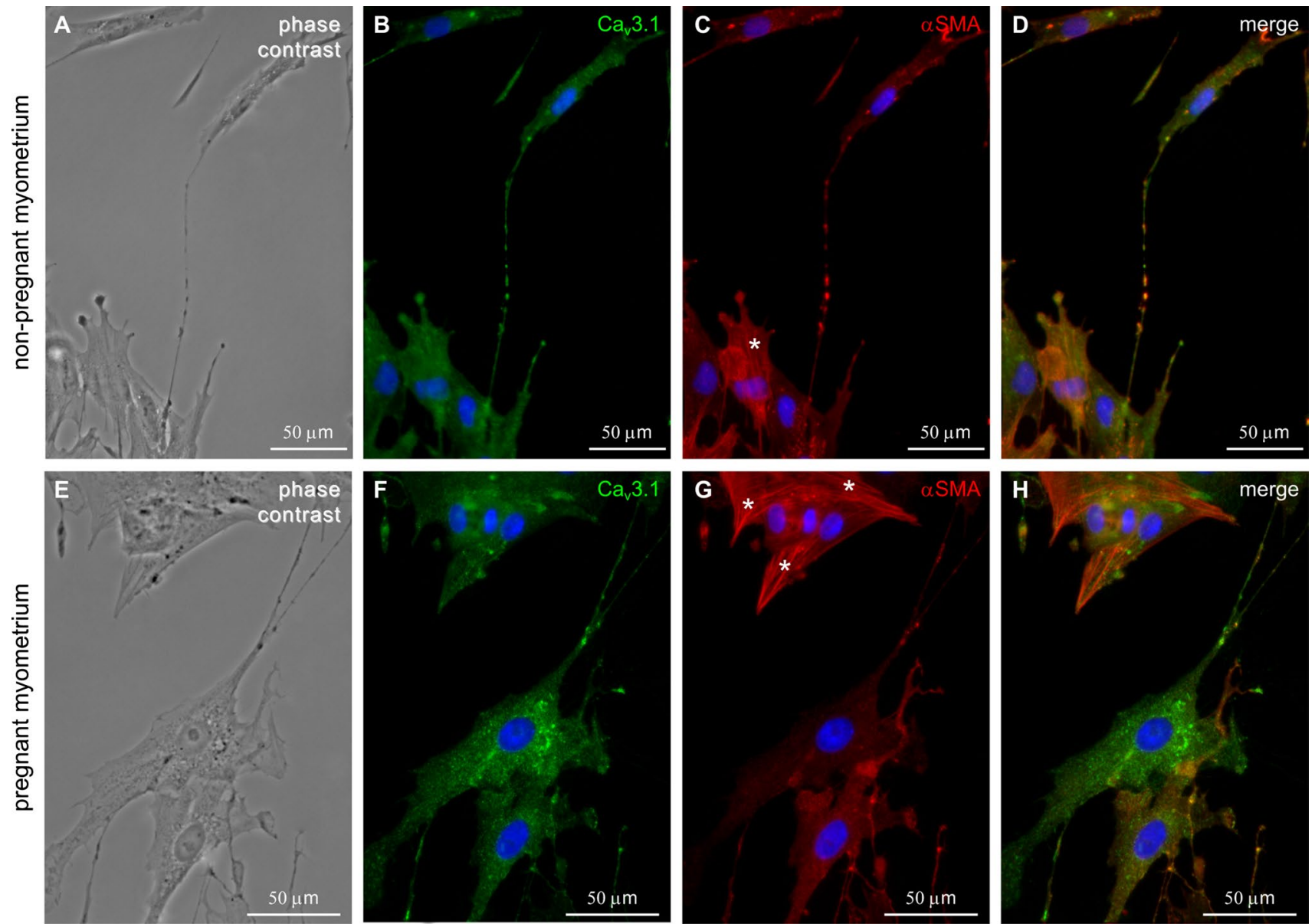

Fig. 4 Double immunolabeling for $\mathrm{Ca}_{\mathrm{V}} 3.1$ and $\alpha$-SMA in cell cultures from non-pregnant/pregnant myometrium. a, e Phase contrast. b, f Strong staining for $\mathrm{Ca}_{\mathrm{V}} 3.1$ was found on both cell body and Tps with widened bodies and without cellular extensions (asterisks)]. d, h Merged images of $\mathrm{Ca}_{\mathrm{V}} 3.1$ and $\alpha$-SMA. Nuclei were stained with DAPI. Bar $50 \mu \mathrm{m}$ 
Fig. 5 Electrophysiological activity recorded in TCs from non-pregnant myometrium. a TC visualized by transmitted light microscopy in phase-contrast illumination mode during patch-clamp recording. b TCs generated no detectable voltageactivated calcium currents when tested with a step depolarization protocol. The same protocol was applied on $n=12$ TCs. Inset Step depolarization protocol of $100 \mathrm{~ms}$ duration from -90 to $+40 \mathrm{mV}$ incremented by $10-\mathrm{mV}$ steps, from a holding potential of $-110 \mathrm{mV}$. $\mathbf{c}$ Representative T-type calcium current and HVA current in a TC (black line) and the blocking effect of $1 \mu \mathrm{M}$ mibefradil on $\mathrm{Ca}^{2+}$ currents (red line). Inset Ramp commands of 100 ms duration from -90 to $+60 \mathrm{mV}$ in voltage-clamp mode. d Current amplitude of T-type and HVA calcium currents in control and mibefradil exposure conditions expressed as mean \pm SD $(n=4)$
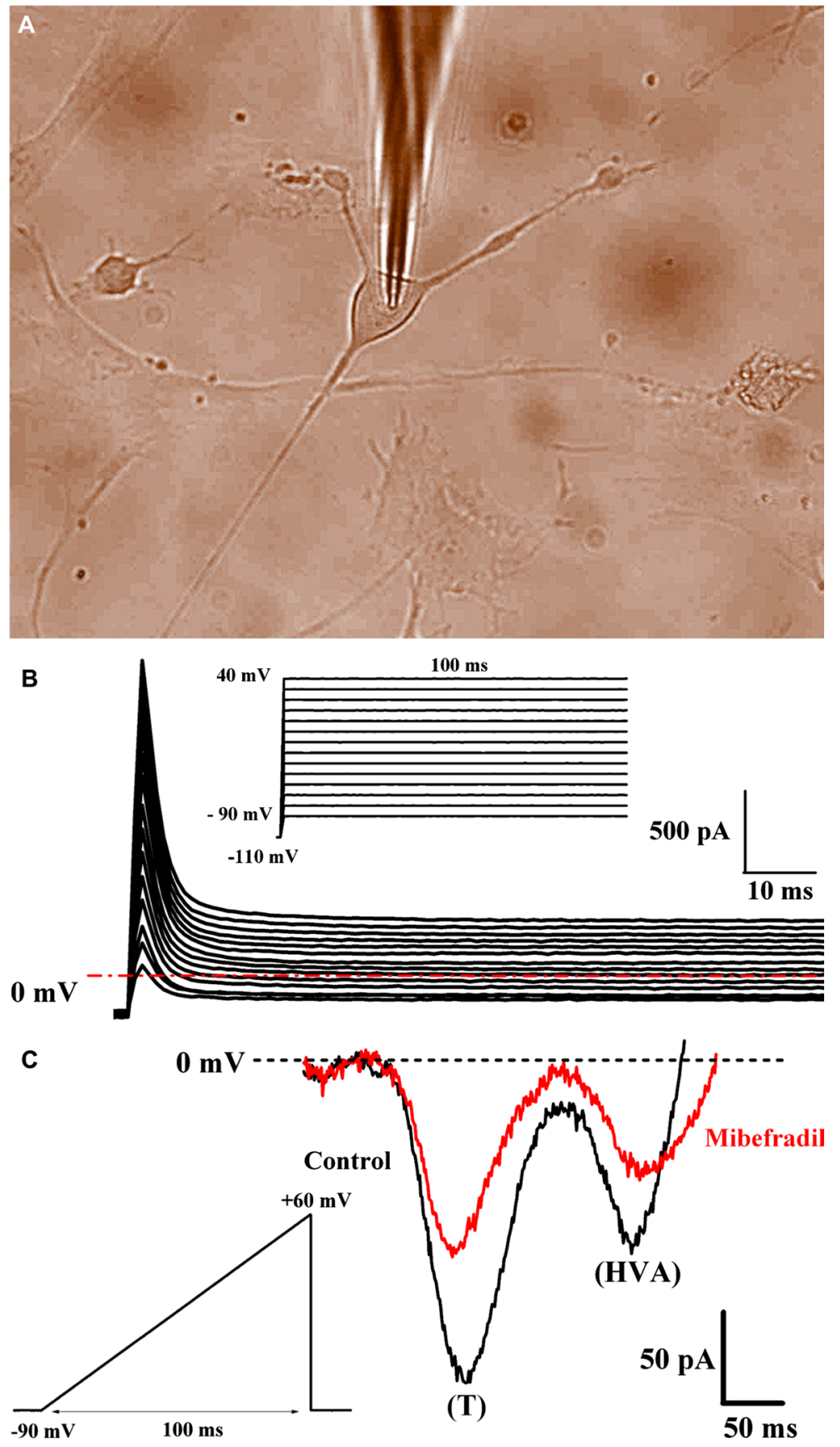

D

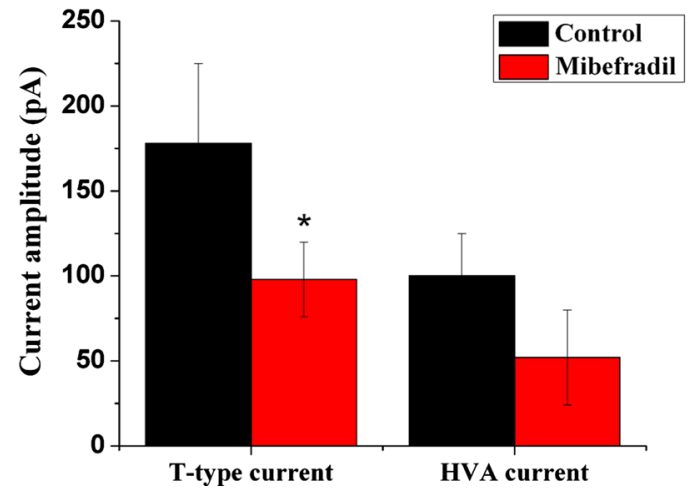


The influence of pregnancy on T-type calcium channel expression in human myometrium is still to be understood. $\mathrm{Ca}_{\mathrm{V}} 3.1$ and $\mathrm{Ca}_{\mathrm{V}} 3.2$ mRNA levels in human myometrial SMCs do not change significantly depending on gestation or labor status (Blanks et al. 2007). On the other hand, different human $\alpha_{1 \mathrm{H}}$ isoforms resulted from alternative splicing in III-IV linker of $\mathrm{Ca}_{\mathrm{V}} 3.2$ have been predominantly evidenced in non-pregnant uterus with respect to pregnant uterus (Ohkubo et al. 2005). Our study brings the first evidence on $\mathrm{Ca}_{\mathrm{V}} 3.1$ and $\mathrm{Ca}_{\mathrm{V}} 3.2$ expression in TCs from human non-pregnant and pregnant myometrium and highlights differences in localization and level of expression of these proteins due to the pregnancy status.

Further confirmation of T-type calcium channels functionality in TCs from non-pregnant myometrium was done by recording inward currents activated by a brief ramp depolarization protocol that were significantly reduced by mibefradil $(1 \mu \mathrm{M})$. In fact, mibefradil has been previously described as a strong antagonist of T-type calcium currents (Clozel et al. 1997), which inhibits the bioelectrical signal and uterine contractile forces in human myometrial tissue strips (Young and Zhang 2005).

Previous studies have reported step depolarization protocols as ineffective for activating voltage-gated calcium currents in cardiac progenitor cells (Tufan et al. 2012) or TCs from non-pregnant myometrium (Cretoiu et al. 2013), meanwhile evidencing the presence of these currents by brief ramp depolarization protocols (Tufan et al. 2012). Therefore, the 'absence' of inward currents previously reported in interstitial cells from myometrium (Duquette et al. 2005) can be correlated with the use of classical step depolarization protocols.

Differences of electrophysiological parameters (e.g., membrane capacitance, input resistance, membrane resting potential) between uterine TCs and SMCs have been previously reported (Duquette et al. 2005). Compared to the two cellular subpopulations described for uterine SMCs, one population presenting HVA currents and a second population (55\%) having both HVA and LVA currents (Blanks et al. 2007), our study has evidenced that TCs have both types of currents. As the currents in SMCs are evoked by 'classical' step depolarization protocols (Blanks et al. 2007), and we show that TCs are responsive only to ramp depolarization protocol and not to step depolarization protocols, it is difficult to exclude the existence of other TCs subpopulations with only HVA currents. Moreover, the steady-state activation/inactivation described for the SMCs population expressing both HVA and LVA currents (Blanks et al. 2007) is absent in TCs presenting both currents.

The presence of T-type calcium channels in uterine TCs might be the missing link for describing the molecular mechanisms by which TCs are involved in mechanical stretching during uterine enlargement in pregnancy. TCs proteomic analysis revealed the up-regulation of myosin-14 (Zheng et al. 2014a) known to be involved in sensory perception (Zong et al. 2012). Periplakin, a protein which links cytoskeleton elements in between and to the junctional complexes, was also found to be upregulated in TCs (Zheng et al. 2014a). Mechanical junctions are indispensable for the proliferation, migration and transformation of different cell types (Leung et al. 2002). Based on previous evidence regarding the existence of heterocellular junctions between TCs and SMC (Cretoiu et al. 2013) and taking into account the existence of cytoskeleton elements (e.g., myosin-14, periplakin), we can hypothesize that TCs could be responsible for detecting the SMC stretch during their enlargement in pregnancy. Moreover, TCs are sometimes described as vimentin-positive cells (Duquette et al. 2005; Zheng et al. 2012), and it was shown that vimentin intermediate filaments determine the mechanical behavior in many cell types (Wang and Stamenovic 2002). Therefore, considering our recent study proving different mechanical reactivity to low-level 1,064-nm laser stimulation of the telopodal lateral extension in non-pregnant versus pregnant myometrium preparations (Campeanu et al. 2014), we propose that TCs play a role of stretch sensor in human myometrium. TCs might be capable of detecting and translate stretch information to the nucleus, determining the production and release of extracellular vesicles and modifying the extracellular homeostasis. These events might also be influenced by steroid hormones, since previous studies showed the existence of estrogen and progesterone receptors in TCs (Cretoiu et al. 2006, 2009). Moreover, we have to emphasize the fact that TCs reactivity to low-level 1,064-nm laser stimulation was influenced by mibefradil (Campeanu et al. 2014) that could imply the contribution of T-type calcium channels in TCs to their capacity as a stretch sensor in human myometrium.

In fetal cardiac myocytes, T-type $\mathrm{Ca}^{2+}$ channels were suggested to play an important role in the regulation of cardiomyocyte size. After birth, T-type $\mathrm{Ca}^{2+}$ channels are involved in the exit of cardiomyocytes from the cell cycle (Wang et al. 2013). Also, there are suggestive studies about existing linkage between $\mathrm{Ca}^{2+}$ influx through T-type $\mathrm{Ca}^{2+}$ channels and the cell proliferation (Taylor et al. 2008). In addition, it seems that in vascular smooth muscle cells, T-type channels might ensure a feedback mechanism to hold off excessive vasoconstriction, being versatile players especially in pathophysiological situations (Kuo et al. 2014). Therefore, there is a possibility that T-type $\mathrm{Ca}^{2+}$ channels could be only transiently present in TCs. They could influence the regulation of myometrial tissue remodeling-hyperplasia in the first phase and stretch-induced myometrial hypertrophy accompanied by lack of uterine contractions during the second half of pregnancy. Our hypothesis is in concordance with recent 
data showing that TCs are involved in tissue morphogenesis during development (Zheng et al. 2014a) and play a part in regeneration and aging (Zheng et al. 2014b). Edelstein and Smythies explored the possibility that TCs could be players in a model system based on bioelectrical signaling (Edelstein and Smythies 2014), and probably, this could be applied for human uterus. Considering that $\mathrm{Ca}_{\mathrm{v}} 3$ T-type calcium channel isoforms differentially distribute to somatic and dendritic compartments in central neurons (McKay et al. 2006), it is not surprising to observe our quantitative immunofluorescence data pointing out the more prevalent distribution of $\mathrm{Ca}_{\mathrm{V}} 3.1$ and $\mathrm{Ca}_{\mathrm{v}} 3.2$ in the telopodes compared to the cell bodies of TCs. Although we already have found a correlation between the elongation of telopodes lateral extensions and T-type calcium channels in uterine TCs (Campeanu et al. 2014), a determined role of these channels in TCs 'bioelectrical signaling' is still to be understood.

For the moment, the subfamily of T-type (transient) calcium channels $\mathrm{Ca}_{\mathrm{v}} 3.1$ and $\mathrm{Ca}_{\mathrm{v}} 3.2$ presence, on the cell body and Tps of TCs in cell cultures obtained from nonpregnant and pregnant myometrium, might represent the starting point to understand the nature of the molecular mechanism responsible for the generation of spontaneous contractions in the myometrium. In our opinion, it is possible that both TCs and SMCs share some of the signaling machinery used for excitation. The differences found in T-type calcium channels subtypes expression in TCs might suggest that T-type $\mathrm{Ca}^{2+}$ channels may be involved in the initiation of action potentials in myometrium at TCs level and then continued at SMCs level. Taking into account that in the last 10 years, a plethora of functions was revealed for the T-type $\mathrm{Ca}^{2+}$ channels, especially in the regulation of critical body functions, and that these channels are able to interact with other ion channels, membrane proteins or bioactive lipids (Nilius and Carbone 2014), we can hypothesize that the differences in expression might be under the pregnancy special hormonal environment. Therefore, steroid hormones and also oxytocin might influence T-type $\mathrm{Ca}^{2+}$ channels higher expression (and not only) in TCs derived from pregnant myometrium, since it is known that TCs have steroid hormone receptors. These might lead to frequent and sustained contractions able to trigger birth. Some other hypothesis might consist of the possibility that the presence of T-type $\mathrm{Ca}^{2+}$ channel calcium signals on TCs might contribute to cell migration and proliferation of myometrial tissue (Rodman et al. 2005) and not point to a pacemaker role.

In conclusion, our data provide immunocytochemical and electrophysiological demonstration of $\mathrm{Ca}_{\mathrm{v}} 3.1$ and $\mathrm{Ca}_{\mathrm{v}} 3.2$ channels expression in uterine TCs. Further functional studies are needed to investigate the function of these channels in non-pregnant and pregnant myometrium as well as in pathological uterine conditions.

Acknowledgments We are grateful to Mrs. Cornelia Dragomir and Mr. T Regalia for their expert technical assistance. This project was conducted through the program Partnerships in priority areas-PN II, developed with the support of ANCS, CNDI-UEFISCDI, Project No. 82/2012. This paper is partly supported (for DD Banciu) by the Sectorial Operational Programme Human Resources Development (SOPHRD), financed by the European Social Fund and the Romanian Government under the contract number POSDRU/159/1.5/S/141531. This work was partially supported by Italian ministerial fellowship awarded to Dr. BM Radu by the D.M. 198/2003.

Conflict of interest The authors confirm that there is no conflict of interest.

Open Access This article is distributed under the terms of the Creative Commons Attribution License which permits any use, distribution, and reproduction in any medium, provided the original author(s) and the source are credited.

\section{References}

Aguilar HN, Mitchell BF (2010) Physiological pathways and molecular mechanisms regulating uterine contractility. Hum Reprod Update 16:725-744

Baruscotti M, DiFrancesco D, Robinson RB (2000) Na(+) current contribution to the diastolic depolarization in newborn rabbit SA node cells. Am J Physiol Heart Circ Physiol 279:H2303-H2309

Bassotti G, Villanacci V, Creţoiu D, Creţoiu SM, Becheanu G (2013) Cellular and molecular basis of chronic constipation: taking the functional/idiopathic label out. World J Gastroenterol 19:4099-4105

Blanks AM, Zhao ZH, Shmygol A, Bru-Mercier G, Astle S, Thornton $S$ (2007) Characterization of the molecular and electrophysiological properties of the T-type calcium channel in human myometrium. J Physiol 581:915-926

Burgess A, Vigneron S, Brioudes E, Labbé J-C, Lorca T, Castro A (2010) Loss of human Greatwall results in G2 arrest and multiple mitotic defects due to deregulation of the cyclin B-Cdc2/PP2A balance. Proc Natl Acad Sci USA 107:12564-12569

Campeanu RA, Radu BM, Cretoiu SM, Banciu DD, Banciu A, Cretoiu D, Popescu LM (2014) Near infrared low-level laser stimulation of telocytes from human myometrium. Lasers Med Sci. doi:10.1007/s10103-014-1589-1

Ceafalan L, Gherghiceanu M, Popescu LM, Simionescu O (2012) Telocytes in human skin - are they involved in skin regeneration? J Cell Mol Med 16:1405-1420

Chen X, Zheng Y, Manole CG, Wang X, Wang Q (2013) Telocytes in human oesophagus. J Cell Mol Med 17:1506-1512

Ciontea SM, Radu E, Regalia T, Ceafalan L, Cretoiu D, Gherghiceanu M, Braga RI, Malincenco M, Zagrean L, Hinescu ME, Popescu LM (2005) c-kit immunopositive interstitial cells (Cajal-type) in human myometrium. J Cell Mol Med 9:407-420

Clozel JP, Ertel SI, Ertel EA (1997) Discovery and main pharmacological properties of mibefradil (Ro 40-5967), the first selective T-type calcium channel blocker. J Hypertens 15:S17-S25

Comunanza V, Carbone E, Marcantoni A, Sher E, Ursu D (2011) Calcium-dependent inhibition of T-type calcium channels by TRPV1 activation in rat sensory neurons. Pflug Arch Eur J Physiol 462:709-722 
Corradi LS, Jesus MM, Fochi RA, Vilamaior PS, Justulin LA Jr, Góes RM, Felisbino SL, Taboga SR (2013) Structural and ultrastructural evidence for telocytes in prostate stroma. J Cell Mol Med 17:398-406

Cretoiu D, Ciontea SM, Popescu LM, Ceafalan L, Ardeleanu C (2006) Interstitial Cajal-like cells (ICLC) as steroid hormone sensors in human myometrium: immunocytochemical approach. J Cell Mol Med 10:789-795

Cretoiu SM, Cretoiu D, Suciu L, Popescu LM (2009) Interstitial Cajal-like cells of human Fallopian tube express estrogen and progesterone receptors. J Mol Histol 40:387-394

Cretoiu SM, Simionescu AA, Caravia L, Curici A, Cretoiu D, Popescu LM (2011) Complex effects of imatinib on spontaneous and oxytocin-induced contractions in human non-pregnant myometrium. Acta Physiol Hung 98:329-338

Cretoiu D, Cretoiu SM, Simionescu AA, Popescu LM (2012a) Telocytes, a distinct type of cell among the stromal cells present in the lamina propria of jejunum. Histol Histopathol 27:1067-1078

Cretoiu SM, Cretoiu D, Popescu LM (2012b) Human myometriumthe ultrastructural 3D network of telocytes. J Cell Mol Med 16:2844-2849

Cretoiu SM, Cretoiu D, Marin A, Radu BM, Popescu LM (2013) Telocytes: ultrastructural, immunohistochemical and electrophysiological characteristics in human myometrium. Reproduction 145:357-370

Deguchi S, Ohashi T, Sato M (2006) Tensile properties of single stress fibers isolated from cultured vascular smooth muscle cells. J Biomech 39:2603-2610

Díaz-Flores L, Gutiérrez R, Sáez FJ, Díaz-Flores L Jr, Madrid JF (2013) Telocytes in neuromuscular spindles. J Cell Mol Med 17:457-465

Duquette RA, Shmygol A, Vaillant C, Mobasheri A, Pope M, Burdyga T, Wray S (2005) Vimentin-positive, c-kit-negative interstitial cells in human and rat uterus: a role in pacemaking? Biol Reprod 72:276-283

Edelstein L, Smythies J (2014) The role of telocytes in morphogenetic bioelectrical signaling: once more unto the breach. Front Mol Neurosci 7:41

Galiger C, Kostin S, Golec A, Ahlbrecht K, Becker S, Gherghiceanu M, Popescu LM, Morty R, Seeger W, Voswinckell R (2014) Phenotypical and ultrastructural features of Oct4-positive cells in the adult mouse lung. J Cell Mol Med. doi:10.1111/jcmm.12295

Gherghiceanu M, Popescu LM (2011) Heterocellular communication in the heart: electron tomography of telocyte-myocyte junctions. J Cell Mol Med 15:1005-1011

Gherghiceanu M, Popescu LM (2012) Cardiac telocytes - their junctions and functional implications. Cell Tissue Research 348:265-279

Hutchings G, Williams O, Cretoiu D, Ciontea SM (2009) Myometrial interstitial cells and the coordination of myometrial contractility. J Cell Mol Med 13:4268-4282

Kuo IY, Howitt L, Sandow SL, McFarlane A, Hansen PB, Hill CE (2014) Role of T-type channels in vasomotor function: team player or chameleon? Pflüg Archiv Eur J Physiol 466:767-779

Leoni P, Carli F, Halliday D (1990) Intermediate filaments in smooth muscle from pregnant and non-pregnant human uterus. Biochem J 269:31-34

Leung CL, Green KJ, Liem RK (2002) Plakins: a family of versatile cytolinker proteins. Trends Cell Biol 12:37-45

Leuranguer V, Mangoni ME, Nargeot J, Richard S (2001) Inhibition of T-type and L-type calcium channels by mibefradil: physiologic and pharmacologic bases of cardiovascular effects. J Cardiovasc Pharmacol 37:649-661

Luesma MJ, Gherghiceanu M, Popescu LM (2013) Telocytes and stem cells in limbus and uvea of mouse eye. J Cell Mol Med 17:1016-1024
Matsumoto T, Nagayama K (2012) Tensile properties of vascular smooth muscle cells: bridging vascular and cellular biomechanics. J Biomech 45:745-755

Matyja A, Gil K, Pasternak A, Sztefko K, Gajda M, Tomaszewski KA, Matyja M, Walocha JA, Kulig J, Thor P (2013) Telocytes: new insight into the pathogenesis of gallstone disease. J Cell Mol Med 17:734-742

McKay BE, McRory JE, Molineux ML, Hamid J, Snutch TP, Zamponi GW, Turner RW (2006) $\mathrm{Ca}(\mathrm{V}) 3$ T-type calcium channel isoforms differentially distribute to somatic and dendritic compartments in rat central neurons. Eur J Neurosci 24: 2581-2594

Milia AF, Ruffo M, Manetti M, Rosa I, Conte D, Fazi M, Messerini L, Ibba-Manneschi L (2013) Telocytes in Crohn's disease. J Cell Mol Med 17:1525-1536

Mosher AA, Rainey KJ, Bolstad SS, Lye SJ, Mitchell BF, Olson DM, Wood SL, Slater DM (2013) Development and validation of primary human myometrial cell culture models to study pregnancy and labour. BMC Pregnancy Childbirth Suppl 1(S1):S7

Mou Y, Wang Y, Li J, Lü S, Duan C, Du Z, Yang G, Chen W, Zhao S, Zhou J, Wang C (2013) Immunohistochemical characterization and functional identification of mammary gland telocytes in the self-assembly of reconstituted breast cancer tissue in vitro. J Cell Mol Med 17:65-75

Nilius B, Carbone E (2014) Amazing T-type calcium channels: updating functional properties in health and disease. Pflug Arch 466:623-626

Ohkubo T, Inoue Y, Kawarabayashi T, Kitamura K (2005) Identification and electrophysiological characteristics of isoforms of T-type calcium channel $\mathrm{Ca}(\mathrm{v}) 3.2$ expressed in pregnant human uterus. Cell Physiol Biochem 16:245-254

Popescu LM, Faussone-Pellegrini MS (2010) TELOCYTES-a case of serendipity: the winding way from Interstitial Cells of Cajal (ICC), via Interstitial Cajal-Like Cells (ICLC) to TELOCYTES. J Cell Mol Med 14:729-740

Popescu LM, Nicolescu MI (2013) Telocytes and stem cells. In: dos Santos Goldenberg RC, Campos de Carvalho AC (eds) Resident stem cells and regenerative therapy. Academic Press, Oxford, pp 205-231

Popescu LM, Gherghiceanu M, Cretoiu D, Radu E (2005) The connective connection: interstitial cells of Cajal (ICC) and ICC-like cells establish synapses with immunoreactive cells. Electron microscope study in situ. J Cell Mol Med 9:714-730

Popescu LM, Gherghiceanu M, Hinescu ME, Cretoiu D, Ceafalan L, Regalia T, Popescu AC, Ardeleanu C, Mandache E (2006a) Insights into the interstitium of ventricular myocardium: interstitial Cajal-like cells (ICLC). J Cell Mol Med 10:429-458

Popescu LM, Vidulescu C, Curici A, Caravia L, Simionescu AA, Ciontea SM, Simion S (2006b) Imatinib inhibits spontaneous rhythmic contractions of human uterus and intestine. Eur J Pharmacol 546:177-181

Popescu LM, Ciontea SM, Cretoiu D (2007) Interstitial Cajal-like cells in human uterus and fallopian tube. Ann N Y Acad Sci 1101:139-165

Qi G, Lin M, Xu M, Manole CG, Wang X, Zhu T (2012) Telocytes in the human kidney cortex. J Cell Mol Med 16:3116-3122

Radu BM, Dumitrescu DI, Marin A, Banciu DD, Iancu AD, Selescu T, Radu M (2014) Advanced type 1 diabetes is associated with ASIC alterations in mouse lower thoracic dorsal root ganglia neurons. Cell Biochem Biophys 68:9-23

Rodman DM, Reese K, Harral J, Fouty B, Wu S, West J, Hoedt-Miller M, Tada Y, Li KX, Cool C, Fagan K, Cribbs L (2005) Low-voltage-activated (T-type) calcium channels control proliferation of human pulmonary artery myocytes. Circ Res 96:864-872

Rosenbaum ST, Svalø J, Nielsen K, Larsen T, Jørgensen JC, Bouchelouche P (2012) Immunolocalization and expression of 
small-conductance calcium-activated potassium channels in human myometrium. J Cell Mol Med 16:3001-3008

Senadheera S, Bertrand PP, Grayson TH, Leader L, Tare M, Murphy TV, Sandow SL (2013) Enhanced contractility in pregnancy is associated with augmented TRPC3, L-type, and T-type voltagedependent calcium channel function in rat uterine radial artery. Am J Physiol Regul Integr Compar Physiol 305:R917-R926

Sheng J, Shim W, Lu J, Lim SY, Ong BH, Lim TS, Liew R, Chua YL, Wong P (2014) Electrophysiology of human cardiac atrial and ventricular telocytes. J Cell Mol Med 18:355-362

Sun X, Zheng M, Zhang M, Qian M, Zheng Y, Li M, Cretoiu D, Chen C, Chen L, Popescu LM, Wang X (2014) Differences in the expression of chromosome 1 genes between lung telocytes and other cells: mesenchymal stem cells, fibroblasts, alveolar type II cells, airway epithelial cells and lymphocytes. J Cell Mol Med 18:801-810

Taylor JT, Zeng XB, Pottle JE, Lee K, Wang AR, Yi SG, Scruggs JA, Sikka SS, Li M (2008) Calcium signaling and T-type calcium channels in cancer cell cycling. World J Gastroenterol 14:4984-4991

Tufan H, Zhang XH, Haghshenas N, Sussman MA, Cleemann L, Morad M (2012) Cardiac progenitor cells engineered with Pim-1 (CPCeP) develop cardiac phenotypic electrophysiological properties as they are co-cultured with neonatal myocytes. J Mol Cell Cardiol 53:695-706

Vannucchi MG, Traini C, Manetti M, Ibba-Manneschi L, FaussonePellegrini MS (2013) Telocytes express PDGFR $\alpha$ in the human gastrointestinal tract. J Cell Mol Med 17:1099-1108

Wang N, Stamenovic D (2002) Mechanics of vimentin intermediate filaments. J Muscle Res Cell Motil 23:535-540

Wang F, Gao H, Kubo H, Fan X, Zhang H, Berretta R, Chen X, Sharp T, Starosta T, Makarewich C, Li Y, Molkentin JD, Houser SR
(2013) T-type $\mathrm{Ca}^{2+}$ channels regulate the exit of cardiac myocytes from the cell cycle after birth. J Mol Cell Cardiol 62:122-130

Xiao J, Wang F, Liu Z, Yang C (2013) Telocytes in liver: electron microscopic and immunofluorescent evidence. J Cell Mol Med 17:1537-1542

Young RC, Zhang P (2005) Inhibition of in vitro contractions of human myometrium by mibefradil, a T-type calcium channel blocker: support for a model using excitation-contraction coupling, and autocrine and paracrine signaling mechanisms. J Soc Gynecol Investig 12:e7-e12

Young RC, Smith LH, McLaren MD (1993) T-type and L-type calcium currents in freshly dispersed human uterine smooth muscle cells. Am J Obstet Gynecol 169:785-792

Zheng Y, Bai C, Wang X (2012) Telocyte morphologies and potential roles in diseases. J Cell Physiol 227:2311-2317

Zheng Y, Zhang M, Qian M, Wang L, Cismasiu VB, Bai C, Popescu LM, Wang X (2013) Genetic comparison of mouse lung telocytes with mesenchymal stem cells and fibroblasts. J Cell Mol Med 174:567-577

Zheng Y, Cretoiu D, Yan GQ, Cretoiu SM, Popescu LM, Wang X (2014a) Comparative proteomic analysis of human lung telocytes with fibroblasts. J Cell Mol Med 18:568-589

Zheng Y, Cretoiu D, Yan G, Cretoiu SM, Popescu LM, Fang H, Wang $\mathrm{X}$ (2014b) Protein profiling of human lung telocytes and microvascular endothelial cells using iTRAQ quantitative proteomics. J Cell Mol Med 18:1035-1059

Zong L, Lu C, Zhao Y, Li Q, Han D, Yang W, Shen Y, Zheng Q, Wang $\mathrm{Q}$ (2012) Clue to a new deafness gene: a large Chinese nonsyndromic hearing loss family linked to DFNA4. J Genet Genomics 39:653-657 